\title{
Ventricular Fibrillation in a Patient with Multi-Vessel Coronary Spasm Four Days after the Initiation of an Oral Beta-blocker
}

\author{
Manabu Kurabayashi, Hidetoshi Suzuki, Tsukasa Shimura, Yasuteru Yamauchi, Kaoru Okishige \\ Division of Cardiology, Yokohama City Minato Red Cross Hospital, Yokohama City, Japan
}

\begin{abstract}
Received: $13 / 05 / 2016$
Accepted: 26/05/2016

Published: 06/07/2016 four days after the initiation of an oral beta-blocker. EJCRIM 2016;3:doi:10.12890/2016_000439

Conflicts of Interests: The Authors declare that there are no competing interests.

This article is licensed under a Commons Attribution Non-Commercial 4.0 License
\end{abstract}

How to cite this article: Kurabayashi M, Suzuki H, Shimura T, Yamauchi Y, Okishige K. Ventricular fibrillation in a patient with multi-vessel coronary spasm

\section{ABSTRACT}

We describe a case of ventricular fibrillation occurring in a patient with multi-vessel coronary spasm after the initiation of an oral betablocker. A 56-year-old man began to experience chest discomfort and his computed tomography revealed intermediate coronary stenoses. He was administered medications including an oral beta-blocker but suddenly collapsed while walking 4 days later. An automated external defibrillator detected ventricular fibrillation and delivered successful electrical cardioversion. An acetylcholine provocation test after stabilization of the status revealed triple-vessel coronary spasm. Beta-blockers may provoke exacerbation of coronary spasm and result in lethal arrhythmia.

\section{LEARNING POINTS}

- Beta-blockers which have a vasoconstrictive effect may occasionally provoke exacerbation of coronary spasm.

- Coronary spasm should be considered as a cause of lethal ventricular arrhythmia or cardiac arrest.

\section{KEYWORDS}

Coronary spasm, ventricular fibrillation, beta-blocker, acetylcholine provocation test.

\section{INTRODUCTION}

Beta-blockers have a vasoconstrictive effect and may provoke exacerbation of coronary spasm ${ }^{[1]}$. It is known that coronary spasm can cause serious arrhythmias and consequently sudden cardiac death ${ }^{[2]}$. We herein describe a case of ventricular fibrillation occurring in a patient with multi-vessel coronary spasm 4 days after the initiation of an oral beta-blocker.

\section{CASE REPORT}

A 56-year-old man with a history of dyslipidaemia began to experience chest discomfort on effort and at rest. He presented to a local hospital where coronary computed tomography revealed intermediate stenoses in the mid portion of the left anterior descending artery (LAD) and the first diagonal branch. The patient was prescribed medications including low-dose aspirin, a statin and a beta-blocker (atenolol 25 mg once daily), but his symptoms worsened.

Four days later he took his medications and went shopping on foot. At $10 \mathrm{am}$, he suddenly collapsed while walking. Cardiac massage was immediately performed by bystanders and after $5 \mathrm{~min}$ of resuscitation effort, an automated external defibrillator (AED) was attached. It detected ventricular fibrillation and delivered a successful electrical cardioversion (Fig. 1A). The AED electrocardiogram (ECG) record just 
after successful cardioversion showed ST-segment elevation (Fig. 1B), which disappeared promptly (Fig. 1C). After the return of spontaneous circulation, the patient was brought to the emergency department of our hospital.

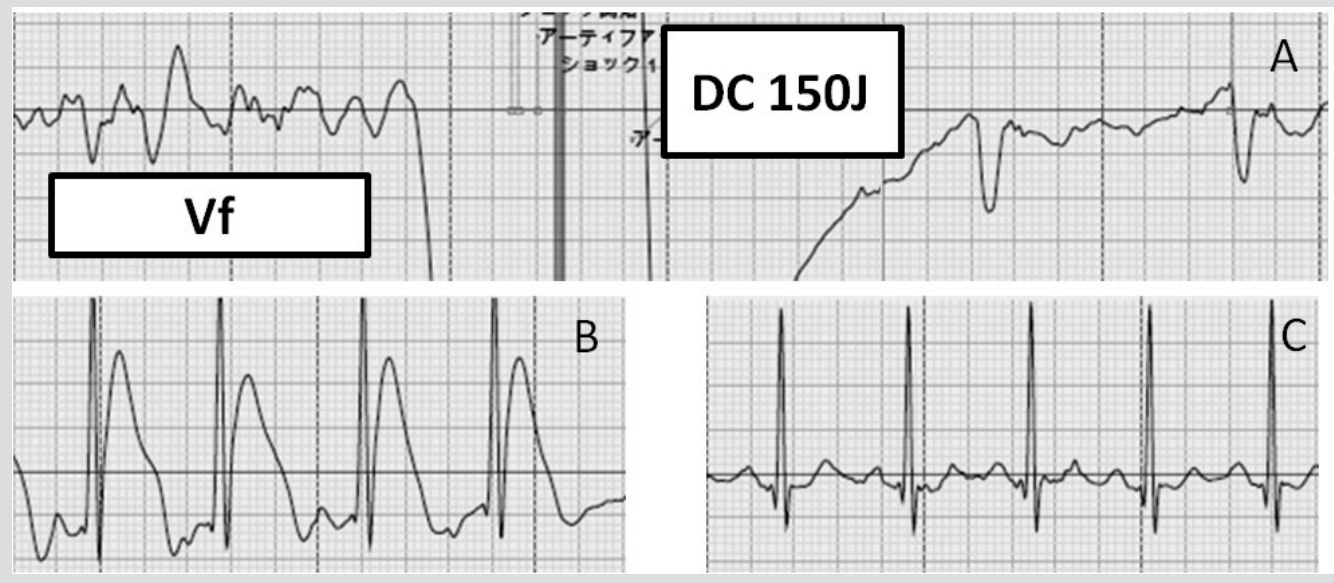

Figure 1: The electrocardiogram record of the automated external defibrillator

At presentation to the emergency department, his ECG and chest x-ray showed almost normal findings. Laboratory analysis revealed normal values for complete blood cell counts, electrolytes and cardiac biomarkers. We performed emergency coronary angiography (CAG) (Fig. $2 A-C$ ), which showed intermediate stenoses in the mid portion of the LAD and the first diagonal branch (Fig. 2B, black arrow) and normal coronary flow in each coronary artery. Based on the cardiac catheterization findings, we ruled out acute coronary syndrome due to coronary plaque rupture as the cause of the ventricular fibrillation. After CAG, we performed intensive care including targeted temperature management and intravenous injection of nicorandil for the prevention of coronary spasm. Following treatment, the patient regained a stable cardiopulmonary status and had no serious ventricular arrhythmias. His neurological status gradually improved and he did not experience any residual neurological deficits.

Eight days after the episode of ventricular fibrillation, we performed an acetylcholine provocation test for coronary spasm after a $48 \mathrm{~h}$ drug holiday from vasodilators. After baseline coronary angiography (Fig. 2D-F), intracoronary injection of $20 \mu \mathrm{g}$ of acetylcholine into the right coronary artery (RCA) and left coronary artery (LCA) provoked coronary spasm of the RCA (Fig. 2G, black arrow head), LAD (Fig. 2H, white arrow head) and left circumflex coronary artery (LCX) (Fig.2l, black arrow). Next, we measured fractional flow reserve, which was 0.85 for the distal LAD, and 0.92 for the first diagonal branch. The patient was diagnosed as having multi-vessel coronary spasm, and the intermediate stenotic lesions of the LAD and the first diagonal branch were not considered to be significant. We prescribed an oral calcium antagonist (nifedipine sustained released tablet, $20 \mathrm{mg}$ twice daily). Fifteen days after the ventricular fibrillation event, we performed a cardiac electrophysiological study while the patient was on an oral calcium antagonist. Programmed ventricular stimulation did not induce reproducible ventricular arrhythmia even with triple extra-stimuli from the right ventricular apex and outflow tract. We therefore attributed the patient's cardiopulmonary arrest to coronary spasm, and continue to prescribe an oral calcium antagonist. Since the initiation of calcium antagonist, the patient has been free from any angina symptoms and ventricular arrhythmias.

\section{DISCUSSION}

Beta-blockers reduce heart rate and contractility, resulting in relief of myocardial ischaemia. However, blockade of the vasodilatory $\beta_{2}{ }^{-}$ adrenergic effect even with $\beta_{1}$-selective agents ${ }^{[3]}$ and relative enhancement of the vasoconstrictive $\alpha 1$-adrenergic effect ${ }^{[1]}$ can result in exacerbation of coronary spasm and provoke lethal arrhythmia. In this case, the patient had experienced ventricular fibrillation 4 days after the initiation of an oral $\beta_{1}$-selective adrenergic blocker, and was ultimately diagnosed as having multi-vessel coronary spasm. The fact that multi-vessel spasm was provoked by intracoronary injection of low-dose acetylcholine indicates that this patient's baseline coronary artery tone may be high. To our knowledge, there are few cases of the initiation of oral beta-blocker therapy resulting in ventricular fibrillation in patients with coronary spastic angina. 
Coronary spasm can cause critical ischaemic heart disease. In particular, multi-vessel spasm is often complicated by lethal ventricular arrhythmias ${ }^{[4]}$, similar to our case. A previous study reported that reperfusion after coronary spasm, rather than coronary spasm itself, correlated with the onset of ventricular arrhythmia in some cases ${ }^{[4]}$. Those patients may not be diagnosed as having coronary spasm following emergency coronary angiography just after resuscitation. Physicians should perform a spasm provocation test after the patient has stabilized and emergency coronary angiography had not revealed any likely cardiac lesions, because coronary spasm was detected in $11 \%$ of patients who survived cardiac arrest without structural heart disease ${ }^{[5]}$. Coronary spasm is a more common cause of lethal ventricular arrhythmia or cardiac arrest than one might expect.

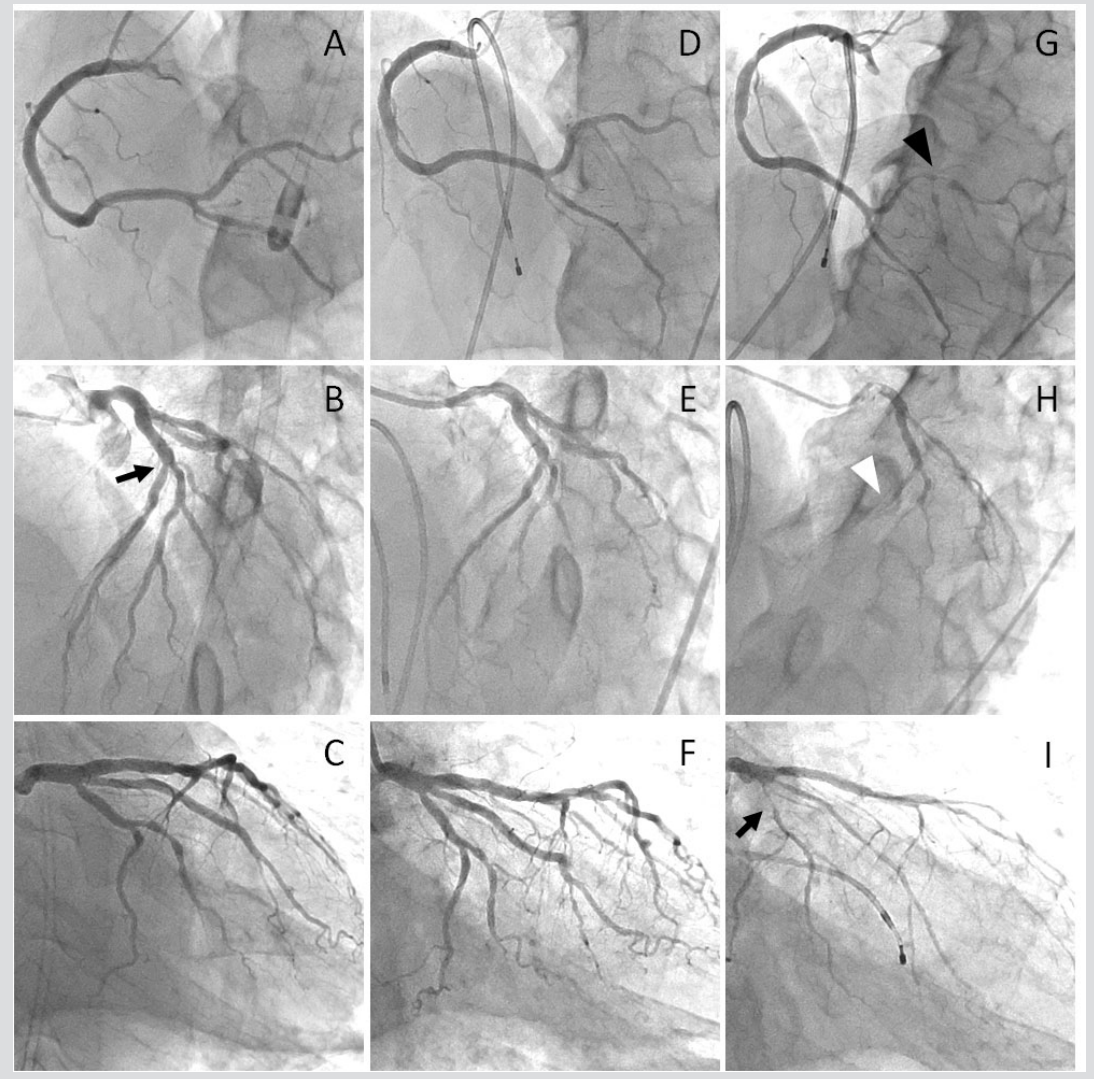

Figure 2: Emergency coronary angiography (A-C) and elective acetylcholine provocation test (D-F: baseline coronary angiography, G-I: acetylcholine provocation test)

\section{REFERENCES}

1. Robertson RM, Wood AJ, Vaughn WK, Robertson D. Exacerbation of vasotonic angina pectoris by propranolol. Circulation 1982;65:281-285

2. Takagi Y, Yasuda S, Tsunoda R, Ogata Y, Seki A, Sumiyoshi T, et al. Clinical characteristics and long-term prognosis of vasospastic angina patients who survived out-of-hospital cardiac arrest: multicenter registry study of the Japanese Coronary Spasm Association. Circ Arrhythm Electrophysiol 2011;4:295-302.

3. Kaplan NM. Kaplan's Clinical Hypertension. 8th ed. Philadelphia: Lippincott Williams \& Wilkins, 2002:261-267.

4. Myerburg RJ, Kessler KM, Mallon SM, Cox MM, deMarchena E, Interian A Jr, et al. Life-threatening ventricular arrhythmias in patients with silent myocardial ischemia due to coronary-artery spasm. N Engl J Med 1992;326:1451-1455.

5. Krahn AD, Healey JS, Chauhan V, Birnie DH, Simpson CS, Champagne J, et al. Systematic assessment of patients with unexplained cardiac arrest: Cardiac Arrest Survivors with Preserved Ejection Fraction Registry (CASPER). Circulation 2009;120:278-285. 